\title{
Dynamical Symmetries of Dirac Hamiltonian
}

\author{
Riazuddin* \\ National Centre for Physics, Quaid-i-Azam University Campus, Islamabad 45320, Pakistan
}

(Dated: November 6, 2018)

\begin{abstract}
Several dynamical symmetries of the Dirac Hamiltonian are reviewed in a systematic manner and the conditions under which such symmetries hold. These include relativistic spin and orbital angular momentum symmetries, $S O(4) \times S U_{\sigma}(2)$ symmetry for the Dirac Hydrogen atom, $S U(3) \times S U_{\sigma}(2)$ symmetry for the relativistic simple harmonic oscillator. The energy spectrum in each case is calculated from group-theoretic considerations.
\end{abstract}

\section{INTRODUCTION}

The $1 / r$ potential of force provides the underlying dynamics for the Kepler problem in classical mechanics, hydrogen atom in quantum mechanics and one gluon exchange potential in perturbative quantum electrodynamics (PQCD). This and simple harmonic oscillator are one of the few physical systems that we know how to solve. Further most of more complicated systems can be studied by representing them as a collection of harmonic oscillators with different frequencies and amplitudes. Not only the above two systems are solvable exactly both in classical and quantum mechanics, they also provide the realistic models to study dynamical symmetries as distinct from geometrical symmetries. Symmetries do play an important role in the progress of physics. Once they are known for a physical system, many of the properties of that system can be established in general terms without actually solving for the underlying dynamics.

As stated above symmetries are of two types: (i) Geometric, the well known example of which are space-time symmetries, e.g. rotation (ii) Dynamical, where the underlying dynamics manifest some symmetry. This is well illustrated both by the hydrogen atom and harmonic oscillator, which are rotationally invariant but also have a well known feature that their orbits of motion close on themselves in classical mechanics. This shows that in addition to angular momentum (conservation which is due to rotational invariance), there are additional constants of motion. For the hydrogen atom this constant of motion, as is well known, is provided by the Rung-Lenz's vector [1]. For the harmonic oscillator this is provided by quadrupole moment second rank tensors [2].

*Electronic address: riazuddin@ncp.edu.pk 
For the hydrogen atom in Schrodinger theory, energy levels are given by $E_{n}=-\frac{m \alpha}{2 n^{2}}$, where $n$ is the principle quantum number, and $\alpha$ is the fine structure constant. For a given $l, n \geq l+1$; $l=0,1,2, \ldots . n$. The $(2 l+1)$ degeneracy with respect to magnetic quantum number is due to rotational symmetry of the potential $-\frac{\alpha}{r}$. For one energy value $E$, there are $\sum_{l=0}^{n-1}(2 l+1)=n^{2}$ different possible eigen-functions; such a degeneracy occurs only for $1 / r$ potential of force. Usually a degeneracy is associated with a symmetry and it is known [1] that in this case there is an external symmetry $[\vec{R}, H]=0$, with $H=\frac{\vec{p}^{2}}{2 m}-\frac{\alpha}{r}$, and $\vec{R}=\frac{1}{2 m}(\vec{p} \times \vec{L}-\vec{L} \times \vec{p})-\frac{\alpha}{r} \vec{r}$. The operator $\vec{R}$ given above in Quantum Mechanics follows from the Lenz's vector in the classical Kepler problem by the correspondence principle. The orbital angular momentum $\vec{L}$ and $\vec{K}=\sqrt{\frac{-m}{2 H}} \vec{R}(\vec{K}$ is hermitian when acting on eigenstates of $H$ with-negative energy eigenvalues- the ones in which we are interested; $\sqrt{\frac{-m}{2 H}}$ commutes with $\vec{R}$ and $\vec{L}$ ) generate $S O(4)$ algebra, which is isomorphic to $S U_{M}(2) \times S U_{N}(2)$ generated by $\vec{M}=\frac{\vec{L}+\vec{K}}{2}, \vec{N}=\frac{\vec{L}-\vec{K}}{2}$. This symmetry leads to $n^{2}$ degeneracy [1] .

Similarly for the non- relativistic harmonic oscillator, the energy spectrum shows degeneracies in addition to those which arise due to rotational invariance. As is well known, the energy spectrum $E_{N}=\frac{1}{2} \hbar w(2 N+3)$ depends on the oscillator quantum number $N=2 n+l$, where $n \geq 0$ is the radial quantum number and $l$ is the orbital angular momentum. Thus all states with $l=N, N-2, \ldots .0$ or 1 have the same energy. These degeneracies are produced by an $S U(3)$ dynamical symmetry [3].

Obviously the above symmetries are broken in the relativistic quantum mechanics, where a spin $1 / 2$ particle satisfies the Dirac equation. As is well known this is because the spin-orbit coupling leads to splitting of the energy levels [see below]. Since spin is purely a quantum mechanical concept with no classical analogue, the guidance from the corresponding principle is not there to obtain dynamical symmetries for the Dirac Hamiltonian. As such it is highly non trivial to discuss under which conditions the larger symmetries are obtained in the Dirac Hamiltonian. This has been answered for harmonic oscillator in [4] and following the method of [4], for the Hydrogen atom in [5]. The purpose of this paper is to review the previous work mentioned above and to discuss the symmetries of the Dirac Hamiltonian in a systematic way, using the Dirac algebra and for harmonic Oscillator $S U(3)$ algebra generated in the Gell-Mann basis [6] and point out which physical system might have such symmetries. In fact we first show that the harmonic oscillator provides a simple realization of $S U(3)$, which has been used in particle and nuclear physics. The energy spectrum in each case has also been calculated from group-theoretic considerations. 


\section{DYNAMICAL SPIN AND ORBITAL ANGULAR MOMENTUM SYMMETRY FOR THE DIRAC HAMILTONIAN}

The Dirac Hamiltonian is given by

$$
H=\vec{\alpha} \cdot \vec{p}+V_{V}(\vec{r})+\beta m
$$

where for the Hydrogen atom $V_{V}(\vec{r})=-\frac{\alpha}{r}$ and is the time component of electromagnetic potential $A^{\mu}(\vec{r}),(\mu=0,1,2,3)$. The hydrogen atom in Dirac theory is exactly solvable [7, 8] and energy levels are given by

$$
E_{n j}=m\left[1+\frac{\alpha^{2}}{\left(n-\delta_{j}\right)^{2}}\right]^{-1 / 2}
$$

where

$$
\delta_{j}=\left(j+\frac{1}{2}\right)-\left[\left(j+\frac{1}{2}\right)^{2}-\alpha^{2}\right]^{1 / 2}
$$

and $j=0,1 / 2,1 \ldots$. The above expression manifests the fine structure and spin-orbital splitting. The states with same $n$ but different $j$ e.g. $2 p_{1 / 2}$ and $2 p_{3 / 2}$ are now split and the splitting is in agreement with experiment, a great triumph of the Dirac theory. The states with same $n$ and $j$ e.g. $2 s_{1 / 2}$ and $2 p_{1 / 2}$ are still degenerate, the so called Lamb shift which needs radiative quantum corrections provided by quantum electrodynamics (QED). The SO(4) symmetry for the hydrogen atom in Schrodinger theory is thus broken in relativistic theory. Usually in the non-relativistic limit i.e to order $\left(p^{2} / m^{2}\right)$, the relativistic theory reduces to non-relativistic theory but in this case the non-relativistic theory has a higher symmetry, namely, $\mathrm{SO}(4)$ which is not maintained by the relativistic theory. The question then arises under what circumstances for hydrogen-like system (by this we mean systems with $1 / r$ force potential), the $\mathrm{SO}(4)$ symmetry is restored for the Dirac Hamiltonian, although it will be contrary to experimental observations for the hydrogen atom. Then why should even this question be considered? This is because there are other physical systems which show such an approximate symmetry as we will discuss shortly. To answer the above question, let us introduce a Lorentz scalar potential $V_{S}(\vec{r})$, then the Dirac Hamiltonian becomes

$$
H=\vec{\alpha} \cdot \vec{p}+V_{V}(\vec{r})+\beta\left(V_{S}(\vec{r})+m\right)
$$

and the corresponding Dirac equation is

$$
\left[i \gamma^{0}\left(\partial_{0}+i V_{v}(r)\right)+i \gamma^{i} \partial_{i}-m-V_{s}(r)\right] \Psi=0
$$


where $\gamma^{i}=\beta \alpha^{i}, \gamma^{0}=\beta$

If we multiply on the left by

$$
\left[i \gamma^{0}\left(\partial_{0}+i V_{V}(r)\right)+i \gamma^{j} \partial_{j}+m+V_{S}(r)\right]
$$

Then for stationary states $\left(\frac{\partial}{\partial t} \rightarrow i E\right)$, the Dirac equation becomes

$$
\left[\nabla^{2}+V_{V}^{2}-V_{S}^{2}-2 E V_{V}-2 m V_{S}+i \gamma^{0} \gamma^{i}\left[\partial_{i}, V_{V}\right]-i \gamma^{i}\left[\partial_{i}, V_{S}\right]+\left(E^{2}-m^{2}\right)\right] \Psi=0
$$

where

$$
\begin{aligned}
{\left[\partial_{i}, V_{V}\right] } & =\frac{\partial V}{\partial x^{i}} \\
{\left[\partial_{j}, V_{S}\right] } & =\frac{\partial V}{\partial x^{j}} .
\end{aligned}
$$

Thus if

$$
\begin{aligned}
& V_{V}(\vec{r})=V(\vec{r})+U_{V} \\
& V_{S}(\vec{r})=V(\vec{r})+U_{S}
\end{aligned}
$$

where $U_{V}$ and $U_{S}$ are constants, then since in the non-relativistic limit $\gamma^{0} \rightarrow 1+O\left(\frac{p^{2}}{m^{2}}\right), \gamma^{i} \rightarrow$ $1+O\left(\frac{p^{i}}{m}\right), E \rightarrow \epsilon+m$, the terms of order $\frac{|p|}{m}$ cancel out in Eq. (5). As a consequence absorbing the constants $U_{V}$ and $U_{S}$ with re-definition of $E$ and $m$, the above equation reduces to the Schrodinger equation, which as already seen has $S O(4)$ symmetry, if $V$ is spherically symmetric.

Indeed it has been observed that the Dirac Hamiltonian (3) is invariant under a spin symmetry 9], $[H, \vec{S}]=0$, provided that conditions (6) or (7) are satisfied. Here the generators $\vec{S}$ form the spin $S U(2)$ algebra and are given by

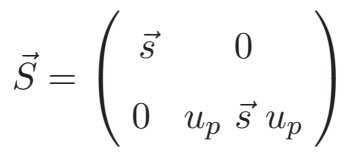

where $\vec{s}=\vec{\sigma} / 2$ are usual spin generators and $u_{p}=\frac{\vec{\sigma} \cdot \vec{p}}{p}$ is the helicity unitary operator. It is easy to check that

$$
\left[S_{i}, S_{j}\right]=i \epsilon_{i j k} S_{k}
$$

In the Pauli representation of Dirac matrices,

$$
\beta=\left(\begin{array}{cc}
1 & 0 \\
0 & -1
\end{array}\right), \quad \vec{\alpha}=\left(\begin{array}{cc}
0 & \vec{\sigma} \\
\vec{\sigma} & 0
\end{array}\right), \quad \gamma^{5}=-i \alpha^{1} \alpha^{2} \alpha^{3}=\left(\begin{array}{cc}
0 & 1 \\
1 & 0
\end{array}\right)
$$


By introducing $\vec{\Sigma}=\left(\begin{array}{cc}\vec{\sigma} & 0 \\ 0 & \vec{\sigma}\end{array}\right)$, one can write the Dirac Hamiltonian (3) and the spin operator (8) as

$$
\begin{gathered}
H=\gamma^{5} \beta \vec{\Sigma} \cdot \vec{p}+V_{V}(\vec{r})+\beta\left(V_{S}(\vec{r})+m\right) \\
\vec{S}=\frac{1}{2}\left[\beta \vec{\Sigma}+(1-\beta) \vec{\Sigma} \cdot \vec{p} \vec{p} \frac{1}{p^{2}}\right]
\end{gathered}
$$

Then, since $[\beta, 1-\beta]=0, \quad[\beta, \vec{\Sigma}]=0, \quad\left[\gamma^{5}, \vec{\Sigma}\right]=0,\left[\gamma^{5}, \beta\right]_{+}=0$ and $\left[\vec{\Sigma} \cdot \vec{p}, \vec{\Sigma} \cdot \vec{p} p^{i}\right]=0$,

$$
\begin{aligned}
{[H, \vec{S}]=} & \frac{1}{4}\left[\gamma^{5}, \beta\right]\left[\vec{\Sigma} \cdot \vec{p}, \Sigma^{i}\right]_{+}+\frac{1}{4}\left[\gamma^{5},(1-\beta)\right]\left[\vec{\Sigma} \cdot \vec{p}, \frac{\vec{\Sigma} \cdot \vec{p} p^{i}}{p^{2}}\right]_{+} \\
& +\frac{(1-\beta)}{2}\left[V_{V}(\vec{r}),(1-\beta) \frac{\vec{\Sigma} \cdot \vec{p} \vec{p}}{p^{2}}\right]+\frac{\beta(1-\beta)}{2}\left[V_{S}(\vec{r}), \frac{\vec{\Sigma} \cdot \vec{p}}{p^{2}} p^{i}\right] \\
= & \frac{1}{2} \gamma^{5} \beta 2 \delta^{i j} p^{j}-\frac{1}{2} \gamma^{5} \beta 2 p^{i}+\frac{(1-\beta)}{2} \Sigma^{j}\left[\left(V_{V}(\vec{r})-V_{S}(\vec{r})\right), \frac{p^{j} p^{i}}{p^{2}}\right]
\end{aligned}
$$

Thus $[H, \vec{S}]=0$ if $\partial^{i} V_{V}(\vec{r})=\partial^{i} V_{S}(\vec{r})$ or $V_{V}(\vec{r})=V_{S}(\vec{r})+U$ i.e. if the conditions (6) or $(7)$ are satisfied. Further for spherically symmetric potentials, $V_{V}(\vec{r})=V_{V}(r), V_{S}(\vec{r})=V_{S}(r)$, the Dirac Hamiltonian has an additional invariant algebra $[9],[H, \vec{L}]=0$ where

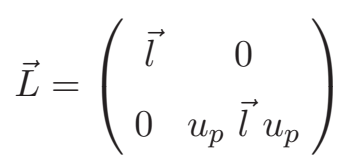

and $\vec{l}=\vec{r} \times \vec{p}$ is the orbital angular momentum. One can write (14) as

$$
\vec{L}=\vec{l}+\frac{(1-\beta)}{2}\left[\vec{\Sigma}-\vec{\Sigma} \cdot \vec{p} \vec{p} / p^{2}\right]
$$

Then $\left[V_{V}(r), \vec{l}\right]=0,\left[V_{S}(r), \vec{l}\right]=0$ and it follows as above that

$$
[H, \vec{L}]=0, \quad\left[L_{i}, L_{j}\right]=i \epsilon_{i j k} L_{k}
$$

Thus $\vec{S}$ and $\vec{L}$ are separately constants of motion while $\vec{s}$ and $\vec{l}$ are not, but $\vec{s}+\vec{l}$ is.

Finally the Dirac Hamiltonian, which has the above relativistic dynamical spin and orbital angular momentum symmetries, is

$$
H=\gamma^{5} \vec{\Sigma} \cdot \vec{p}+(1+\beta) V(r)+\beta m
$$

where constant $U$ can be absorbed in the mass term $m$. We note that

$$
(H+m)=\gamma^{5} \vec{\Sigma} \cdot \vec{p}+(1+\beta)(V+m)
$$




$$
H^{2}-m^{2}=\vec{p}^{2}+2(1+\beta) V(m+V)+\gamma^{5}[(1-\beta) V \vec{\Sigma} \cdot \vec{p}+(1+\beta) V \vec{\Sigma} \cdot \vec{p}]
$$

There is another symmetry the so called relativistic pseudo spin and orbital angular momentum symmetry [9], which is obtained from the one considered above by making the $\gamma_{5}$ transformation and $m \rightarrow-m$

$$
\begin{gathered}
H \rightarrow \widetilde{H}=\gamma^{5} H \gamma^{5}=\gamma^{5} \vec{\Sigma} \cdot \vec{p}+(1-\beta) V(r)+\beta m \\
\vec{S} \rightarrow \widetilde{\vec{S}}=\gamma^{5} S \gamma^{5}=\frac{1}{2}\left[-\beta \vec{\Sigma}+(1+\beta) \frac{\vec{\Sigma} \cdot \vec{p} \vec{p}}{p^{2}}\right] \\
\vec{L} \rightarrow \widetilde{\vec{L}}=\vec{l}+\frac{1+\beta}{2}\left[\vec{\Sigma}-\frac{\vec{\Sigma} \cdot \vec{p} \vec{p}}{p^{2}}\right] \\
{[\widetilde{H}, \widetilde{\vec{S}}]=0,[\widetilde{H}, \widetilde{\vec{L}}]=0}
\end{gathered}
$$

The first of Eqs. (20) implies $V_{V}=-V_{S}+$ constant for pseudo-spin symmetry. The pseudo-spin degeneracies have been observed in nuclei[10]. In fact relativistic mean field representations of the nuclear potential do have this property [11] namely $V_{V} \simeq-V_{S}$.

We now consider the question wether the property $V_{V}(\vec{r})=V_{S}(\vec{r})+U$ arises in QCD. In the $(Q \bar{q})$ or $(q \bar{Q})$ bound states meson spectroscopy, where $Q$ is a heavy quark $c$ or $b$ and $q$ is light quark $u, d$, or $s$, the spin-orbital splitting is seen to be suppressed experimentally [12]. In a $(Q \bar{q})$ system, where $Q$ is considered to be infinitely heavy, the spin $\overrightarrow{S_{Q}}$ of the heavy quark is decoupled, it is then natural to combine the angular momentum of light degrees of freedom $\vec{j}=\vec{L}+\overrightarrow{S_{q}}$ with $\overrightarrow{S_{Q}}$ to give $\vec{J}=\vec{j}+\overrightarrow{S_{Q}}$ for the bound $(Q \bar{q})$ system. Thus for the $p$ states we get two multiplets, one with $j=3 / 2$ and other with $j=1 / 2$ which for $Q=c$, and $q=u$ or $d$ are [and similar one for $B$ meson when $Q=b]$

$$
\begin{array}{ll}
l=0 & {\left[D^{*}\left(1^{-}\right), D\left(0^{-}\right)\right]_{j=\frac{1}{2}},} \\
l=1 \quad\left[D_{2}^{*}\left(2^{+}\right), D_{1}\left(1^{+}\right)\right]_{j=\frac{3}{2}}, \\
& \text { and }\left[\mathrm{D}_{1}^{*}\left(1^{+}\right), D_{0}\left(0^{+}\right)\right]_{j=\frac{1}{2}},
\end{array}
$$

The splitting between $j=\frac{3}{2}$ and $j=\frac{1}{2}$ multiplets is due to spin-orbit coupling $\vec{L} \cdot \overrightarrow{S_{q}}$ (as in hydrogen atom) while the hyperfine splitting between the two members of each multiplet arises 
from the Fermi-contact term $\overrightarrow{S_{q}} \cdot \overrightarrow{S_{Q}}$, the spin-orbit coupling term $\left(\overrightarrow{S_{q}}+\overrightarrow{S_{Q}}\right) \cdot \vec{L}$ and the tensor terms. The splitting [12], for $D$ mesons, between $D_{1}^{*}\left(1^{+}\right): 2422.3 \pm 0.6 \mathrm{MeV}$ and $D_{2}^{*}\left(2^{+}\right): 2462.8 \pm 1.0 \mathrm{MeV}$ is $40 \mathrm{MeV}$; for $B$ mesons, between $B_{1}\left(1^{+}\right): 5723.4 \pm 2.0$ and $B_{2}^{*}\left(2^{+}\right): 5743.9 \pm 5$ is $20 \mathrm{MeV}$; for $B_{s_{1}}\left(1^{+}\right): 5829.4 \pm 0.7$ and $B_{s_{2}}\left(2^{+}\right): 5839.7 \pm 0.6$ is $10 \mathrm{MeV}$. We notice that the spin orbit splittings seem to be suppressed. A measure of this suppression [13] is the parameter

$$
r=\frac{p_{3 / 2}-p_{1 / 2}}{\left(4 p_{3 / 2}+2 p_{1 / 2}\right) / 6-s_{1 / 2}}
$$

which for the experimental data shown above is of order 0.07 both for $D$ and $B$ mesons. We also note that suppression generally increases with the increasing mass of $Q$. It has been suggested [13] that the dynamics necessary for the approximate relativistic spin symmetry discussed above in the heavy-light quark system may be possible in QCD.

\section{III. $S O(4) \times S U_{\sigma}(2)$ SYMMETRY FOR A COULOMB LIKE SYMMETRY POTENTIAL}

In this section we discuss the generators of $S O(4)$ symmetry, which the Dirac Hamiltonian (3) possess. Since in the non-relativistic limit $\beta \rightarrow 1+O\left(p^{2} / m^{2}\right)$ and $\gamma^{5} \rightarrow O(p / m)$, the natural generalization of the Lenz's vector in the Schrodinger theory, for the relativistic case is

$$
2 m \vec{R} \rightarrow 2 m \vec{\Gamma}
$$

where

$$
2 m \vec{\Gamma}=(1+\beta) f(r) \vec{r}+\vec{\Lambda}+\left[(1+\beta) \gamma_{5} g(r) \vec{r} \vec{\Sigma} \cdot \vec{p}+(1-\beta) \gamma_{5} \vec{\Sigma} \cdot \vec{p} g(r) \vec{r}\right]
$$

where now

$$
\vec{\Lambda}=\vec{p} \times \vec{L}-\vec{L} \times \vec{p}
$$

$\vec{L}$ is the relativistic orbital angular momentum defined in Eq. (14). Since $H$ involves $\gamma^{5} \vec{\Sigma} \cdot \vec{p}$, therefore $\vec{\Gamma}$ should involve such a term and the second term in square brackets appears to make the operator hermitian. The functions $f(r)$ and $g(r)$ are to be determined from

$$
[H, \vec{\Gamma}]=0
$$

Now $\vec{L}$ commutes with $H$ and also with $V$ and $\beta m$ and therefore it also commutes with $\gamma^{5} \vec{\Sigma} \cdot \vec{p}$, it follows that $\left[\gamma^{5} \vec{\Sigma} \cdot \vec{p}, \vec{\Lambda}\right]=0$ since $\vec{p}$ commutes with $\vec{\Sigma} \cdot \vec{p}$. Thus using $(\vec{\Sigma} \cdot \vec{p})^{2}=p^{2}$,

$$
\begin{aligned}
2 m[H, \vec{\Gamma}]= & \gamma^{5}[\vec{\Sigma} \cdot \vec{p}, f(r) \vec{r}-2(V+M) g(r) \vec{r}]+\gamma^{5} \beta[\vec{\Sigma} \cdot \vec{p}, f(r) \vec{r}-2(V+M) g(r) \vec{r}]_{+} \\
& +(1+\beta)[V, \vec{\Lambda}]+(1+\beta)\left[p^{2}, g(r) \vec{r}\right]
\end{aligned}
$$


The condition (23) gives

$$
f(r)=2(V+m) g(r)
$$

and

$$
(1+\beta)[V, \vec{\Lambda}]+(1+\beta)\left[p^{2}, g(r) \vec{r}\right]=0
$$

Now using Eq. (15) and the fact that $(1+\beta)(1-\beta)=0$,

$$
\begin{aligned}
(1+\beta)[V, \vec{\Lambda}] & =(1+\beta)[V, \vec{p} \times \vec{l}-\vec{l} \times \vec{p}] \\
& =(1+\beta) \frac{i}{r} \frac{\partial V}{\partial r}[\vec{r} \times \vec{l}-\vec{l} \times \vec{r}]
\end{aligned}
$$

On the other hand

$$
\left[p^{2}, g(r) \vec{r}\right]=-2 i\left[g(r)+\frac{\partial g}{\partial r}\right] \vec{p}-\frac{i}{r} \frac{\partial g}{\partial r}[\vec{r} \times \vec{l}-\vec{l} \times \vec{r}]-\frac{1}{r}\left[\frac{\partial}{\partial r}\left(g(r)+r \frac{\partial g}{\partial r}\right)\right] \vec{r}
$$

It is important to point out that so far we have made no commitment to the form of potentials $V(r)$ and $g(r)$. The condition (26) is satisfied if

$$
g(r)=V(r)
$$

and

$$
V(r)+r \frac{\partial V}{\partial r}=0
$$

which gives $V(r)=$ constt. $\frac{1}{r}$ i.e, the Coulomb potential $-\frac{\alpha}{r}$. It is the constraint (26) which forces the relations $(29)$ and as a result $V(r)$ has to be the Coulomb potential. Thus

$$
2 m \vec{\Gamma}=2(1+\beta) V(V+m) \vec{r}+\vec{\Lambda}+\vec{F}
$$

where

$$
\vec{F}=\gamma_{5}[(1-\beta) V \vec{r} \vec{\Sigma} \cdot \vec{p}+\text { h.c. }]
$$

In order to find $\frac{1}{4 m^{2}}\left[\Gamma^{i}, \Gamma^{j}\right]$, we note that

$$
\begin{gathered}
{\left[\Lambda^{i}, \Lambda^{j}\right]=-4 \epsilon^{i j k} p^{2} L^{k}} \\
{\left[(1+\beta)^{2} V(V+m) x^{i}, \Lambda^{j}\right]-i \leftrightarrow j=-4(1+\beta) V(V+2 M) i \epsilon^{i j k} L^{k}}
\end{gathered}
$$


where we have used, $r \frac{\partial V}{\partial r}=-V(r)$ for $V=-\frac{\alpha}{r}$ and that [c.f. Eq. (15)]

$$
(1+\beta) l^{k}=(1+\beta) L^{k}
$$

Further

$$
\begin{gathered}
{\left[\Lambda^{i}, F^{j}\right]-i \leftrightarrow j=-4 i \epsilon^{i j k} \gamma^{5}[(1-\beta) V \vec{\Sigma} \cdot \vec{p}+(1+\beta) \vec{\Sigma} \cdot \vec{p} V] L^{k}} \\
{\left[(1+\beta) V(V+m) x^{i}, F^{j}\right]-i \leftrightarrow j=0} \\
{\left[F^{i}, F^{j}\right]=-4(1+\beta) \epsilon^{i j k} L^{k} V^{2}}
\end{gathered}
$$

Collecting the various terms and using Eq. (19) with $V=-\alpha / r$ we see that

$$
\left[\Gamma^{i}, \Gamma^{j}\right]=-\frac{H^{2}-m^{2}}{m^{2}} i \epsilon^{i j k} L^{k}
$$

Defining $K^{i}=\sqrt{\frac{m^{2}}{m^{2}-H^{2}}} \Gamma^{i}$, we have finally,

$$
\begin{aligned}
{\left[K^{i}, K^{j}\right] } & =i \epsilon^{i j k} L^{k} \\
{\left[K^{i}, L^{j}\right] } & =i \epsilon^{i j k} K^{k} \\
{\left[L^{i}, L^{j}\right] } & =i \epsilon^{i j k} L^{k}
\end{aligned}
$$

which generate $S O(4)$ algebra. Further $S^{i}$ commutes with $L^{j}$ as well as with $H$ and $K^{j}$. Thus defining

$$
\begin{aligned}
& M^{i}=\frac{L^{i}+K^{i}}{2} \\
& N^{i}=\frac{L^{i}-K^{i}}{2}
\end{aligned}
$$

we see that

$$
\begin{aligned}
{\left[M^{i}, M^{j}\right] } & =i \epsilon^{i j k} M^{k} \\
{\left[N^{i}, N^{j}\right] } & =i \epsilon^{i j k} K^{k} \\
{\left[M^{i}, N^{j}\right] } & =0
\end{aligned}
$$

Thus the invariance group for the Dirac Hamiltonian (13) for the Coulomb potential of the hydrogen atom is $S U_{M} \otimes S U_{N} \otimes S U_{\sigma}(2)$, where $S U_{\sigma}(2)$ is the group generated by $S^{i}$ given in Eq. (12).

The energy spectrum can now be easily determined

$$
\Gamma^{2}=\frac{H^{2}-m^{2}}{m^{2}}\left(L^{2}+1\right)+\alpha^{2} \frac{(H+m)^{2}}{m^{2}}
$$


and $\vec{\Gamma} \cdot \vec{L}=\vec{L} \cdot \vec{\Gamma}=0$, implying $\vec{K} \cdot \vec{L}=0$ so that $M^{2}=N^{2}$. In terms of Casimir operator, $M^{2}$, we can write Eq. (39) as

$$
4\left(m^{2}-H^{2}\right) \vec{M}^{2}=H^{2}-m^{2}+\alpha^{2}(H+m)^{2}
$$

Now since $\vec{M}$ obey angular momentum commutation relations, $\vec{M}^{2}$ has eigenvalues $\mathfrak{m}(\mathfrak{m}+1)$, where $\mathfrak{m}$ can take on the values $0,1 / 2,1, \cdots$. It is customary to use $j$ for $\mathfrak{m}$, then Eq. (40), gives the energy eigenvalues

$$
E=m \frac{4 n^{2}-\alpha^{2}}{4 n^{2}+\alpha^{2}}
$$

where $n=2 j+1$, i.e. the energy spectrum is determined only by the principal quantum number $n$ and states in the different $j$ values are degenerate showing no spin-orbit splitting. In fact Eq. (41) gives for $\epsilon=E-m$

$$
\epsilon_{n}=-m \frac{\alpha^{2}}{2 n}+O\left(\alpha^{4}\right)
$$

which agrees with the energy spectrum for hydrogen atom in Schrodinger theory.

The Dirac equation with vector and/or scalar Coulomb like potentials

$$
\begin{aligned}
& V_{V}(r)=-\frac{\alpha_{V}}{r} \\
& V_{S}(r)=-\frac{\alpha_{S}}{r}
\end{aligned}
$$

is exactly solvable [8] and energy spectrum is given by

$$
E=\frac{m}{\alpha_{V}^{2}+\left(n-\delta_{j}\right)^{2}}\left\{-\alpha_{V} \alpha_{S} \pm\left(n-\delta_{j}\right)\left[\alpha_{V}^{2}-\alpha_{S}^{2}+\left(n-\delta_{j}\right)^{2}\right]^{1 / 2}\right\}
$$

where

$$
\delta_{j}=\left(j+\frac{1}{2}\right)-\left[\left(j+\frac{1}{2}\right)^{2}-\left(\alpha_{V}^{2}-\alpha_{S}^{2}\right)\right]^{1 / 2}
$$

For $\alpha_{V}=\alpha_{S}$, this reduces to Eq. (411).

\section{IV. $S U(3)$ SYMMETRY FOR THE RELATIVISTIC HARMONIC OSCILLATOR}

"The career of a young theoretical physicist consists of treating the harmonic oscillator in everincreasing levels of abstraction." - Sidney Coleman

In this section we discuss how harmonic oscillator provides a simple realistic model to introduce $S U(3)$ symmetry [2] in a language which is more familiar. 
In non-relativistic quantum mechanics, the harmonic oscillator Hamiltonian

$$
H=\frac{1}{2 m}\left[p^{2}+m^{2} \omega^{2} r^{2}\right]
$$

which is symmetric in $p \longleftrightarrow x$, commutes with the quadrupole moment operator $Q^{i j},[i, j=1,2,3]$

$$
Q^{i j}=\left[m^{2} \omega^{2}\left(x^{i} x^{j}-\frac{1}{3} \delta^{i j} x^{2}\right)+\left(p^{i} p^{j}-\frac{1}{3} \delta^{i j} p^{2}\right)\right]
$$

Note that, being symmetric in $i$ and $j$ as well as traceless, $Q^{i j}$ has five independent components while the orbital angular momentum $l^{i j}=x^{i} p^{j}-x^{j} p^{i}$, being antisymmetric in $i$ and $j$, has three independent components. In order to go from tensor basis to the Gell-mann basis, we introduce

$$
F_{a}=\left[m^{2} \omega^{2} \bar{x}^{T} \frac{\lambda_{a}}{2} \bar{x}+\frac{i}{2} m \omega\left(\bar{x}^{T} \frac{\lambda_{a}}{2} \bar{p}-\bar{p}^{T} \frac{\lambda_{a}}{2} \bar{x}\right)+\bar{p}^{T} \frac{\lambda_{a}}{2} \bar{p}\right]
$$

where $a=1, \ldots .8$, and $\bar{x}$ and $\bar{p}$ are column matrices [belonging to representation 3 of $S U(3)$ ]

$$
\bar{x}=\left(\begin{array}{c}
x^{1} \\
x^{2} \\
x^{3}
\end{array}\right), \bar{p}=\left(\begin{array}{c}
p^{1} \\
p^{2} \\
p^{3}
\end{array}\right)
$$

The superscript $T$ denotes transpose so that $\bar{x}^{T}$ and $\bar{p}^{T}$ are row matrices. The tensor and Gell-Mann bases are related by

$$
\begin{aligned}
F_{a} & =\frac{1}{2} \sum_{i, j}\left(\lambda_{a}\right)_{i j} F^{i j} \\
F^{i j} & =\sum_{a}\left(\lambda_{a}\right)^{i j} F_{a}
\end{aligned}
$$

$\lambda_{a}$ are $3 \times 3$ Gell-Mann matrices [6]. The decomposition (477) corresponds to $3 \times 3=3+\overline{6}$ where the representation 3 is antisymmetric and corresponds to orbital angular momentum $l^{i j}$ while the representation $\overline{6}$ is symmetric and correspond to the quadropole moment $Q^{i j}$. In particular $F_{2}=m \omega l^{3}, F_{5}=-m \omega l^{2}, F_{7}=m \omega l^{1}$ while $F_{1}, F_{3}, F_{4}, F_{6}$ and $F_{8}$ correspond to $Q^{i j}$ e.g. $F_{1}=$ $m^{2} \omega^{2} x^{1} x^{2}+p^{1} p^{2}=Q_{x}^{12}+Q_{p}^{12}$. Using the commutation relations, $[I$ is $3 \times 3$ unit matrix $]$

$$
\begin{aligned}
{\left[\bar{x}, \bar{p}^{T}\right] } & =i I \\
{\left[\frac{\lambda_{a}}{2}, \frac{\lambda_{b}}{2}\right] } & =i f_{a b c} \frac{\lambda_{c}}{2}
\end{aligned}
$$

we obtain

$$
\left[F_{a}, F_{b}\right]=i f_{a b c} m \omega F_{c}
$$


where $a, b, c=2,5,7$ and this corresponds to

$$
\left[l^{i}, l^{j}\right]=i \epsilon^{i j k} l^{k}
$$

On the other hand for $a, b=1,3,4,6,8$ in Eq. (51), $c$ is restricted to 2,5,7 and $f_{123}=$ $1,\left(f_{147}, f_{246}, f_{257}, f_{345}\right)=1 / 2,\left(f_{156}, f_{367}\right)=-1 / 2,\left(f_{458}, f_{678}\right)=\sqrt{3} / 2$, all others are zero.

Now for the relativistic harmonic oscillator where we have the Dirac Hamiltonian (3), with $V(r)=\frac{1}{2} m \omega^{2} r^{2}$, qaudrupole moment operator takes the form

$$
\Gamma^{i j}=(1+\beta) f(r) Q_{x}^{i j}+Q_{p}^{i j}+\gamma^{5}\left[(1-\beta) g(r) Q_{x}^{i j} \vec{\Sigma} \cdot \vec{p}+(1+\beta) \vec{\Sigma} \cdot \vec{p} g(r) Q_{x}^{i j}\right]
$$

Since $\left[\gamma^{5} \vec{\Sigma} \cdot \vec{p}, Q_{p}^{i j}\right]=0$

$$
\begin{aligned}
{\left[H, \Gamma^{i j}\right]=} & \gamma^{5}\left[\vec{\Sigma} \cdot \vec{p}, f(r) Q_{x}^{i j}-2(V+m) g(r) Q_{x}^{i j}\right]+\gamma^{5} \beta\left[\vec{\Sigma} \cdot \vec{p}, f(r) Q_{x}^{i j}-2(V+m) g(r) Q_{x}^{i j}\right]_{+} \\
& +(1+\beta)\left[V, Q_{p}^{i j}\right]+(1+\beta)\left[p^{2}, g(r) Q_{x}^{i j}\right]
\end{aligned}
$$

Thus $\left[H, \Gamma^{i j}\right]=0$ gives [with $Q_{x}^{i j}=m^{2} \omega^{2}\left(x^{i} x^{j}-\frac{1}{3} \delta^{i j} x^{2}\right)$ and similar expression for $Q_{p}^{i j}$ ]

$$
\begin{aligned}
& f(r)=2(V+m) g(r) \\
& g(r)=\frac{1}{2 m}
\end{aligned}
$$

with $V(r)=\frac{1}{2} M \omega^{2} r^{2}$. Thus

$$
\Gamma^{i j}=(1+\beta) \frac{1}{m}(V+m) Q_{x}^{i j}+Q_{p}^{i j}+\frac{1}{2 m} \gamma^{5}\left[(1-\beta) Q_{x}^{i j} \vec{\Sigma} \cdot \vec{p}+(1+\beta) \vec{\Sigma} \cdot \vec{p} Q_{x}^{i j}\right]
$$

In the Gell-Mann basis this becomes

$$
\Gamma_{a}=(1+\beta) \frac{1}{m}(V+m) F_{a}^{x}+F_{a}^{p}+\frac{1}{2 m} \gamma^{5}\left[(1-\beta) F_{a}^{x} \vec{\Sigma} \cdot \vec{p}+(1+\beta) \vec{\Sigma} \cdot \vec{p} F_{a}^{x}\right]
$$

Then using the commutation relations (50) and (51)

$$
\left[\Gamma_{a}, \Gamma_{b}\right]=i f_{a b c} \omega\left((1+\beta)(V+m) F_{c}+\frac{1}{2} \gamma^{5}\left[(1-\beta) F_{c} \vec{\Sigma} \cdot \vec{p}+(1+\beta) \vec{\Sigma} \cdot \vec{p} F_{c}\right]\right)
$$

where $F_{c}=i m \omega\left[\bar{x}^{T} \frac{\lambda_{c}}{2} \bar{p}-\bar{p}^{T} \frac{\lambda_{c}}{2} \bar{x}\right]$. Thus

$$
\left[\Gamma_{a}, \Gamma_{b}\right]=i f_{a b c} \omega\left[(H+m) F_{c}+\frac{1}{2} \gamma^{5}(1-\beta)\left[F_{c}, \vec{\Sigma} \cdot \vec{p}\right]\right]
$$

where $a, b=1,3,4,6,8$ while $c=2,5,7$, so that $F_{c}$ is essentially the orbital angular momentum $\vec{l}$. Now using Eqs. (15) and (18)

$$
\begin{aligned}
(H+m) L^{k} & =\left[\gamma^{5} \vec{\Sigma} \cdot \vec{p}+(1+\beta)(V+m)\right]\left[l^{k}+\frac{1}{2}(1-\beta)\left(\Sigma^{k}-\frac{\vec{\Sigma} \cdot \vec{p}}{p^{2}} p^{k}\right]\right. \\
& =(H+m) l^{k}+\frac{i}{2} \gamma^{5}(1-\beta)(\vec{\Sigma} \times \vec{p})^{k} \\
& =(H+m) l^{k}+\frac{1}{2} \gamma^{5}(1-\beta) \frac{1}{m \omega}\left[F_{c}, \vec{\Sigma} \cdot \vec{p}\right]
\end{aligned}
$$


where we have used $\frac{1}{m \omega}\left[F_{c}, \vec{\Sigma} \cdot \vec{p}\right] \sim\left[l^{k}, \vec{\Sigma} \cdot \vec{p}\right]=\frac{i}{2}(\vec{\Sigma} \times \vec{p})^{k}$, Thus from Eqs. (60) and (61)

$$
\left[\widetilde{\Gamma}_{a}, \widetilde{\Gamma}_{b}\right]=i f_{a b c} \widetilde{F}_{c}
$$

where now $\widetilde{F}_{2}=L^{3}, \widetilde{F}_{5}=-L^{2}, \widetilde{F}_{7}=L^{1}$ and $\widetilde{\Gamma}_{a}=\frac{\Gamma_{a}}{\sqrt{m \omega^{2}(H+m)}}$. Further

$$
\left[\widetilde{F}_{a}, \widetilde{F}_{b}\right]=i f_{a b c} \widetilde{F}_{c}
$$

$a, b, c=2,5,7$ and

$$
\left[\widetilde{F}_{a}, \widetilde{\Gamma}_{b}\right]=i f_{a b c} \widetilde{\Gamma}_{c}
$$

where $a=2,5,7$ and $b, c=1,3,4,6,8$.

The commutation relations (62, 63, 64) generate the $S U(3)$ algebra. Since $\vec{S}$ commutes with $H$ as well as with all the above generators, the invariant algebra of the relativistic harmonic oscillator represented by the Dirac Hamiltonian (3) with $V(r)=\frac{1}{2} m \omega^{2} r^{2}$ is $S U(3) \otimes S U_{\sigma}(2)$.

We now calculate the energy spectrum for which purpose we note from Eq. (58) that $[a=$ $1,3,4,6,8]$

$$
\Gamma^{2}=\sum_{a} \Gamma_{a} \Gamma_{a}=\frac{1}{3}\left(H^{2}-m^{2}\right)^{2}-m \omega^{2}(H+m)\left(L^{2}+3\right)
$$

where we have used the definitions of $F_{a}^{x}$ and $F_{a}^{p}$ given in Eq. (45), e.g. $F_{1}^{x}=m \omega^{2} x_{1} x_{2}$ and $F_{1}^{p}=p_{1} p_{2}$, Eqs. (11) and (19) with $V=\frac{1}{2} m \omega^{2} r^{2},(1+\beta)(1-\beta)=0, \gamma_{5}(1 \pm \beta)=(1 \mp \beta) \gamma_{5}$ and Eq. (15). In terms of $\widetilde{\Gamma}_{a}$, Eq. (65) takes the form

$$
3 m \omega^{2}\left[\sum_{a} \widetilde{\Gamma}_{a} \widetilde{\Gamma}_{a}+L^{2}\right]=(H-m)^{2}(H+m)-9 m \omega^{2}
$$

But $L^{2}=\sum_{b} \widetilde{F}_{b}^{2}$, where $b=2,5,7$. Thus Eq. (66) takes the form

$$
3 m \omega^{2} \widetilde{\Gamma}^{2}=(H-m)^{2}(H+m)-9 m \omega^{2}
$$

where $\widetilde{\Gamma}^{2}=\sum_{a} \widetilde{\Gamma}_{a} \widetilde{\Gamma}_{a}+\sum_{b} \widetilde{\Gamma}_{b} \widetilde{\Gamma}_{b}$ is the invariant of the group $\mathrm{SU}(3)$ and as such is proportional to unit matrix.

Hence Eq. (67) gives the energy eigenvalues

$$
(E-m)^{2}(E+m)-9 m \omega^{2}=C m \omega^{2}
$$

where $C$ is to be fixed. This can be done in the following way. We take the non-relativistic limit of Eq. (18), $H \rightarrow H_{\text {non-rel }}+m$, which gives

$$
H_{n o n-r e l}=\frac{p^{2}}{2 m}+2 V=\frac{p^{2}}{2 m}+m \omega^{2} r^{2}
$$


which, as is well known, gives the energy eigenvalues [note we have to replace $\omega$ by $\sqrt{2} \omega$ in the ordinary harmonic oscillator eigenvalues]

$$
\epsilon_{N}=\frac{1}{\sqrt{2}} \omega(2 N+3)
$$

Now we take the non-relativistic limit of Eq. (68), $E \rightarrow \mathcal{E}_{N}+m$, which gives

$$
m \omega^{2}(2 N+3)^{2}-9 m \omega^{2}=C m \omega^{2}
$$

fixing $C=4 N(N+3)$. Putting back in Eq. (68) the energy eigenvalue equation becomes

$$
\left(E_{N}-m\right)^{2}\left(E_{N}+m\right)=4\left(N+\frac{3}{2}\right)^{2} m \omega^{2}
$$

where $N=0,1, \cdots$. This agrees with one obtained from the exact solutions of Dirac equation [14].

\section{SUMMARY AND CONCLUSIONS}

We have systematically reviewed the various dynamical symmetries of the Dirac Hamiltonian, clearly stating the conditions under which such symmetries hold. These symmetries include relativistic spin (pseudo-spin) and orbital angular symmetries which hold when Dirac Hamiltonian with scalar $V_{S}(r)$ and vector $V_{V}(r)$ spherically symmetric potentials satisfy $\partial V_{V} / \partial r= \pm \partial V_{S} / \partial r$ or $V_{V}= \pm V_{S}+U$. Here $U$ is a constant potential but can be absorbed in redefinition of the mass. Then if $V(r)=-\alpha / r$, as for the hydrogen atom or in QCD the Dirac Hamiltonian has $S O(4) \otimes S U_{\sigma}(2)$ or equivalently $S U_{M}(2) \otimes S U_{N}(2) \otimes S U_{\sigma}(2)$ symmetry. Here $S U_{\sigma}(2)$ is the $S U(2)$ group generated by the relativistic spin $S^{i}$ defined in Eq. (8) or Eq. (12). If $V(r)=\frac{1}{2} M \omega^{2} r^{2}$, the harmonic oscillator potential, then the symmetry is $S U(3) \otimes S U_{\sigma}(2)$. We have used Dirac algebra of Dirac matrices and for the simple harmonic oscillation, Gell-Mann basis of $S U(3)$, which is more transparent and simple (at least for physicist with particle physics background) compared to the basis used in [4]. We have also calculated energy spectrum in each case from group theoretical consideration, which agrees with the exact solution of Dirac equation in each case. We have also indicated physical systems where dynamical symmetries discussed above are possibly relevant.

[1] G. Baym, Lectures on Quantum Mechanics, The Benjamin/Cremmings Puli. Com. Inc, (1969).

[2] D. M. Fradkin, Three-Dimensional Isotropic Harmonic Oscillator and SU(3), Am. J. Phys. 33, 207-211 (1965). 
[3] J. P. Elliott, Collective Motion in the Nuclear Shell Model. I. Classification Schemes for States of Mixed Configurations, Proc. R. Soc. A 245, 128-145 (1958).

[4] J. N. Ginocchio, $U(3)$ and Pseudo- $U(3)$ Symmetry of the Relativistic Harmonic Oscillator, Phys. Rev. Letters, 95, 252501-1-252501-3 (2005).

[5] F. L. Zhang, B. Fu and J. L. Chen, Dynamical symmetry of Dirac hydrogen atom with spin symmetry and its connection with Ginocchios oscillator, Phys. Rev. A 78, 040101-1-040101-4 (R) (2008).

[6] See for example, Fayyazuddin and Riazuddin, A modern introduction to Particle Physics, 2nd Edition, World Scientific (2000).

[7] See, Claude Itzykson and Jean-Bernard Zuber, Quantum Field Theory, McGraw-Hill International Editions (1980).

[8] W. Greiner, Relativistic Quantum Mechanics, Springer, Berlin (2000); See also Symmetry of Dirac Equation and corresponding phenomenology, Hong-Wei Ke et al. arxiv: 0907.0051, (2009).

[9] J. S. Bell and H. Ruegg, Dirac equations with an exact higher symmetry, Nucl. Phys. B 98, 151-153 (1975); A. L. Blokhin, C. Bahri, and J. P. Draayer, Origin of Pseudospin Symmetry, Phys. Rev. Lett. 74, 4149-4152 (1995); J. N. Ginocchio and A. Leviatan, On the relativistic foundations of pseudospin symmetry in nuclei, Phys. Lett. B 425, 1-5 (1998); J. N. Ginocchio, A relativistic symmetry in nuclei, Phys. Rep. 315, 213-240 (1999).

[10] A. Arima, M. Harrey, and K. Shimizu, Pseudo $L S$ coupling and pseudo $S U_{3}$ coupling schemes, Phys. Lett. B 30, 517-522 (1969); K.Hecht and A. Adlen, Generalized seniority for favored $J \neq 0$ pairs in mixed configurations, Nucl. Physic. A 137, 129-143 (1969).

[11] B. D. Serot and J. D. Walecka, in The Relativistic Nuclear Many-Body Problem in Advances in Nuclear Physics, edited by J.W. Negele and E. Vogt (Plenum, New York, 1986), Vol. 16; B. A. Nikolaus, T. Hoch, and D. G. Madland, Nuclear ground state properties in a relativistic point coupling model, Phys. Rev. C 46, 1757-1781 (1992).

[12] K. Nakamura et al., Review of Particle Physics (Particle Data Group), J. Phys. G 37, 075021 (2010).

[13] P. R. Page, T. Goldman and J. N. Ginocchio, Relativistic Symmetry Suppresses Quark Spin-Orbit Splitting, Phys. Rev. Lett. 86, 204-207 (2001).

[14] J. N. Ginocchio, Relativistic harmonic oscillator with spin symmetry, Phys. Rev. C 69, 034318-1034318-8 (2004). 